\title{
2D - FINITE ELEMENT MODEL OF A CIGS MODULE
}

\author{
G. J.M. Janssen, L. H. Slooff, E.E. Bende \\ ECN Solar Energy, P.O. Box 1, 1755 ZG Petten, The Netherlands, \\ Phone: +31 88515 4803, Fax: +31 88515 8214, E-mail: janssen@ecn.nl
}

\begin{abstract}
The performance of thin-film CIGS modules is often limited due to inhomogeneities in CIGS layers. A 2-dimensional Finite Element Model for CIGS modules is demonstrated that predicts the impact of such inhomogeneities on the module performance. Results are presented of a module with a region of poor diode characteristics. It is concluded that according to this model the effects of poor diodes depend strongly on their location in the module and on their dispersion over the module surface. Due to its generic character the model can also be applied to other series connections of photovoltaic cells.
\end{abstract}

Keywords: CIGS, Finite Element Method, Modeling, Module, Thin-Film Solar Cell

\section{INTRODUCTION}

A substantial gap exists between efficiencies that are achieved in full scale thin-film CIGS modules and efficiencies reported for laboratory-scale cells [1,2]. The efficiency of a thin-film CIGS module is often substantially limited by non-uniformities or defects that occur in the layers deposited on the scale of the full module surface area. Such inhomogeneities may include variations in composition and crystallite sizes, grain boundaries, and stresses. As a result there will be variations of the photo-electrical properties such as the diode saturation current, the contact resistance, the shunt resistance, the photogeneration current, or combinations thereof $[3,4]$. It is clear that these inhomogeneities can extend over more than one cell [5,6]. Experimental techniques such as electroluminescence, photoluminescence or thermography can be used to identify these inhomogeneities in the module.

Simple analytical models will not always be sufficient to predict the effect of these inhomogeneities on the module performance [5-8]. The distributed series resistance of the contact layers implies that an individual CIGS solar cell cannot be described by a set of parallel one-diode equivalent circuits operating at the same voltage [7]. Additionally, the monolithic contact over the full cell length makes that a simple series connection of the individual cells is not always a valid approach.

A model that takes into account the 2D spatial distribution of properties of the inhomogeneities over the module surface is required for an accurate prediction of the performance. This can be implemented by describing the module by a network of 1-diode equivalent circuits that are coupled through the conductive contact layers. Finite element methods (FEM) are a suitable tool to solve the 2D partial differential equations that form the basis of such a model.

In this paper we demonstrate the model for a four-cell CIGS module. However, due to its generic character the model is not limited to CIGS and can be applied to other photovoltaic modules as well. The model can predict the performance at various operation conditions (illumination, temperature, imposed current or voltage). In addition to overall performance data of the module such as $I-V$ characteristics and efficiency, the voltage and current distribution in each cell can be obtained as well as the heat dissipated in the active layer and in the connecting and contact layers. Results will be presented that were obtained for the case of inohomogeneities being represented by a higher diode dark saturation current density $j_{0}$. The results will illustrate the considerable impact that inhomogeneities can have on the module output when they are delocalized over more than one cell.

\section{MODEL}

A CIGS module consists of $n$ cells with width $w$ and length $L$. In the present model each cell is considered to consist of an active layer and two contact layers. The active layer can be a CIGS absorber layer with a CdS buffer layer. The properties of this layer are described by a local diode saturation current density $j_{0}$, an ideality factor $A$, and a shunt resistance $R_{\text {shunt }}$. The contact layers are the Transparent Conductive Oxide layer, e.g. ZnO:Al, and a Mo layer. These layers are characterized by their conductivity $\sigma$ and thickness $t$. An additional contact resistance between the active layer and a contact layer may be included. The monolithic series connection is implemented by connecting the $\mathrm{ZnO}$ :Al layer of one cell to the Mo layer of the adjacent cell, over the whole length of the cell. Although in this model the thickness, length and conductivity of the connecting layer can be specified at will, we apply here a simplified version of the usual configuration of the monolithic CIGS interconnect [8], shown in Figure 1. This means that the conductivity is that of $\mathrm{ZnO}: \mathrm{Al}$. The length of the connect is equal to the thickness of the active layer $d$. The thickness of the connecting layer is $s$, i.e. the width of the scribe made between the active layers. The model is completed by defining a photogeneration current density $j_{L}$.

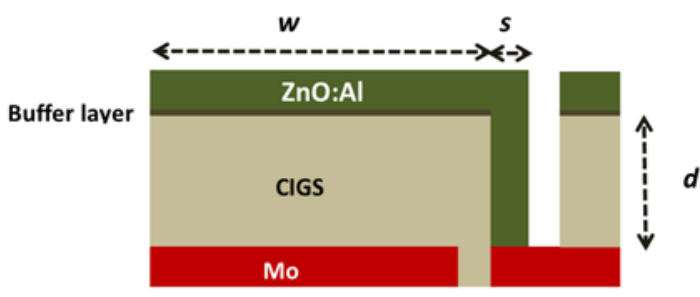

Figure 1: Side view of a CIGS cell and an adjacent cell showing the interconnection as incorporated in the model 


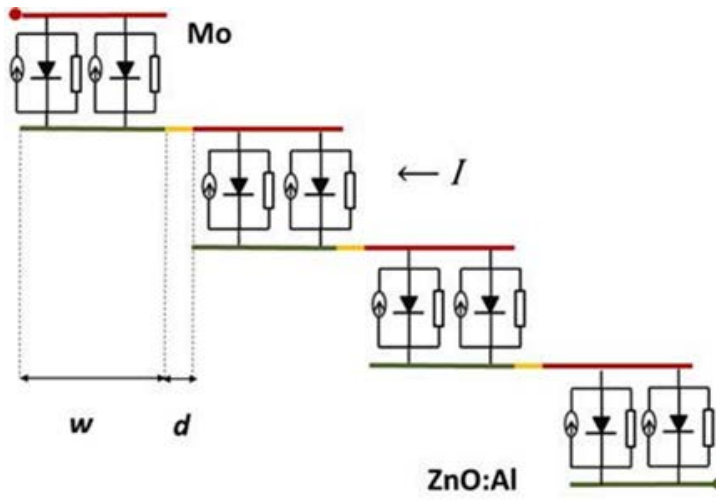

Figure 2: Schematic showing the equivalent circuit representation of four cells connected in series.

A schematic drawing of the model layout for a fourcell module is shown in Figure 2. In this figure the red lines represent Mo layers, the green lines $\mathrm{ZnO}$ :Al layers, and the yellow lines the interconnects. Although only two equivalent circuits are drawn per cell, each cell is in fact represented by a large number of 1-diode circuits, both in the direction of the plane of the paper (cell width) and in the direction perpendicular to the paper (cell length). The module is contacted at the Mo layer of the cell on top of the structure and at the $\mathrm{ZnO}$ :Al layer at the bottom. It is assumed that the contacting is over the full cell length.

As mentioned above, all physical parameters included can be made dependent upon the $(x, y)$ position in the module, i.e. they have a spatial distribution. In the present study only the dark saturation current density was made position dependent. Moreover, no contact resistance was assumed between the active layer and the contact layers. The model solves the distribution of the potential $u$ in the $n+1$ conductive layers shown in Figure 2. Depending on whether the position in the layer corresponds to $\mathrm{Mo}$, to $\mathrm{ZnO}: \mathrm{Al}$, or to the interconnect the equivalent circuits act as sources or sinks of current, or they do not contribute at all (interconnect region). For $n$ cells this approach leads to a set of $n+1$ coupled 2dimensional non-linear Poisson equations for the local potential $u$.

The boundary conditions are those of zero current, i.e. $\nabla u \cdot \mathbf{n}=0$ at all boundaries except where the module is contacted. At these boundaries $u=0$ respectively $u=\mathrm{V}_{\text {module }}$ is imposed. The equations are solved using a Finite Element Method, with a spatial resolution of about 4080 elements per cell. The values for physical parameters used in the calculations are listed in Table I.

\section{RESULTS}

\subsection{Single cell}

In a single cell a 'poor zone' was defined which comprised $40 \%$ of the cell surface area. The values chosen for the dark saturation current density $j_{0}$ in the good and poor parts of the cell correspond to $V_{o c}$ values of $650 \mathrm{mV}$ and $282 \mathrm{mV}$, respectively. This large difference was chosen here to highlight the effect of poor areas, but experimental data show that such variations can indeed be encountered in CIGS modules [8]. The area averaged value of $j_{0}$ results in $2.02 \mu \mathrm{Acm}^{-2}$ with a corresponding $V_{o c}$ of $311 \mathrm{mV}$. Calculations using the
Table I: Parameters used in the calculations

\begin{tabular}{|c|r|c|r|}
\hline Parameter & \multicolumn{1}{|c|}{ Value } & Parameter & \multicolumn{1}{c|}{ Value } \\
\hline$L$ & $10 \mathrm{~cm}$ & $j_{L}$ & $34 \mathrm{mAcm}^{-2}$ \\
\hline$w$ & $0.5 \mathrm{~cm}$ & $A$ & 1.245 \\
\hline$d$ & $2 \mu \mathrm{m}$ & $j_{0}$ good & $50.6 \mathrm{fAcm}^{-2}$ \\
\hline$s$ & $160 \mu \mathrm{m}$ & $j_{0}$ poor & $5.06 \mu \mathrm{Acm}^{-2}$ \\
\hline$t$ ZnO:Al & $1 \mu \mathrm{m}$ & $R_{\text {shunt }}$ & $1 \mathrm{kOhmcm}^{2}$ \\
\hline$t \mathrm{Mo}$ & $1 \mu \mathrm{m}$ & $\sigma \mathrm{ZnO}: \mathrm{Al}$ & $450 \mathrm{Scm}^{-1}$ \\
\hline$T$ & $298.15 \mathrm{~K}$ & $\sigma \mathrm{Mo}$ & $3 \cdot 10^{4} \mathrm{Scm}^{-1}$ \\
\hline
\end{tabular}

parameters in Table I show that the single $5 \mathrm{~cm}^{2}$ cell then has a maximum power output of $27.8 \mathrm{~mW}$, and the corresponding $I-V$ curve has a fill factor of 0.53 . This approach is equivalent to assuming that the $40 \%$ poor area is evenly distributed over the whole cell surface area.

When the $40 \%$ poor area is not distributed over the cell area but localized in a continuous part of the cell, the 2D FEM calculation shows that the maximum power and the $V_{o c}$ increase to, respectively, $29.7 \mathrm{~mW}$ and $352 \mathrm{mV}$, as is also shown in Figure 3. However, the fill factor of the curve with the localized area is reduced to 0.49 .

This is a direct result of the shielding of the poor area that can take place due to finite conductivity of particularly the $\mathrm{ZnO}$ :Al layer. Figure 4 shows the distribution of the dark saturation current density and the resulting distributions of the current density and cell voltage at the maximum power point (MPP). The top figures show the distribution in the case of a homogeneous distribution of poor diode characteristics. The cell voltage and the generated current density vary from left to right as a result of the finite conductivity of contact layers. When there is a localized poor area (bottom figures in Figure 4), the generated current and the cell voltage are reduced in that poor area compared to the good area. In this way, both areas can operate closer to the maximum power point that corresponds to the local diode characteristics.

As a result of the inhomogeneity, the current flow is squeezed to the areas outside the poor zone, which has a negative impact on the fill factor. However, in agreement with the increased maximum power output, the $V_{o c}$ increases in the case of a localized poor area. At open circuit conditions usually current is generated in the areas with good diode characteristics and dissipated in areas

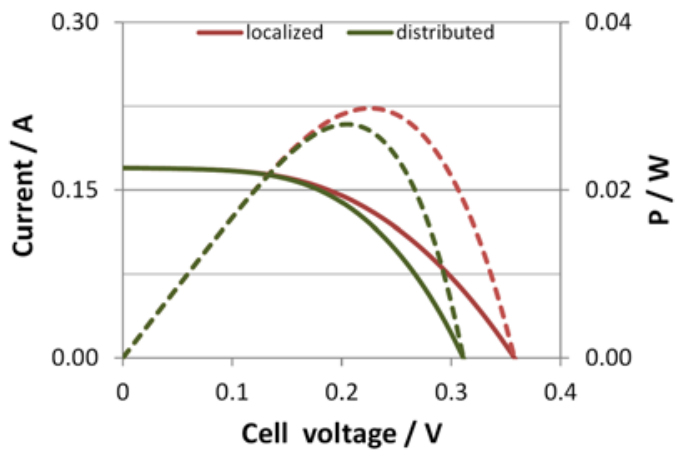

Figure 3: $I-V$ and $P-V$ curve of a cell with $40 \%$ bad area uniformly distributed and as a continuous, isolated area 

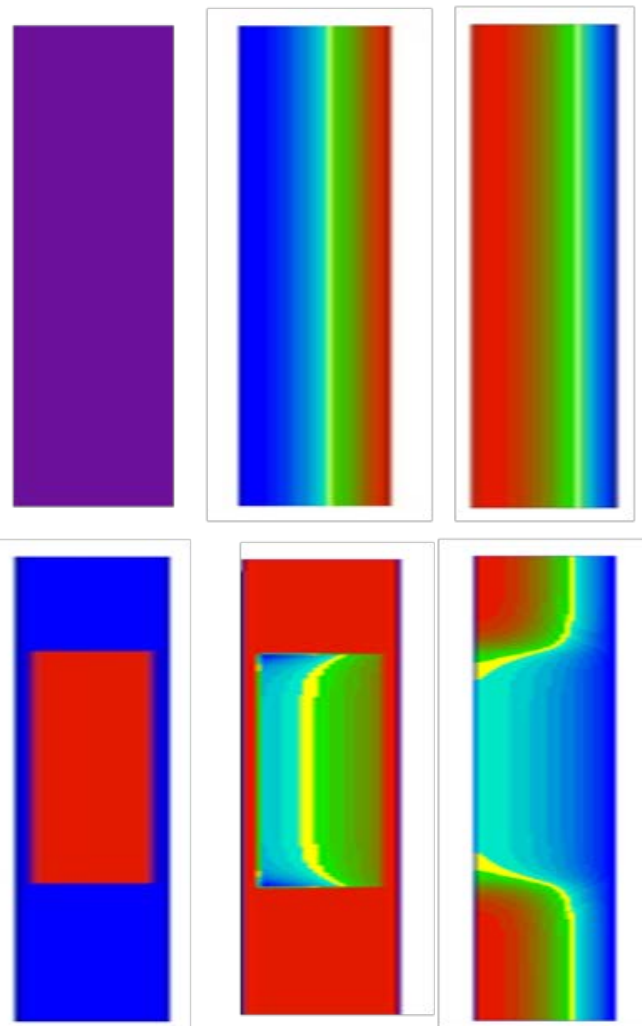

Figure 4: From left to right: the distribution of the dark saturation current density $j_{0}$, of the generated current density at MPP, and of the cell voltage at MPP in a single cell with a bad area. The values increase from blue to red. Top: distributed poor area, bottom localized poor area.

with poor diode characteristics. The finite conductivity enables the good sections to operate at a higher cell voltage than the poor sections, which results in a higher overall $V_{o c}$ value, as illustrated by Figure 5 . Whenever the increase in $V_{o c}$ can outweigh the reduction in fill factor, the maximum power output will increase due to this shielding effect.

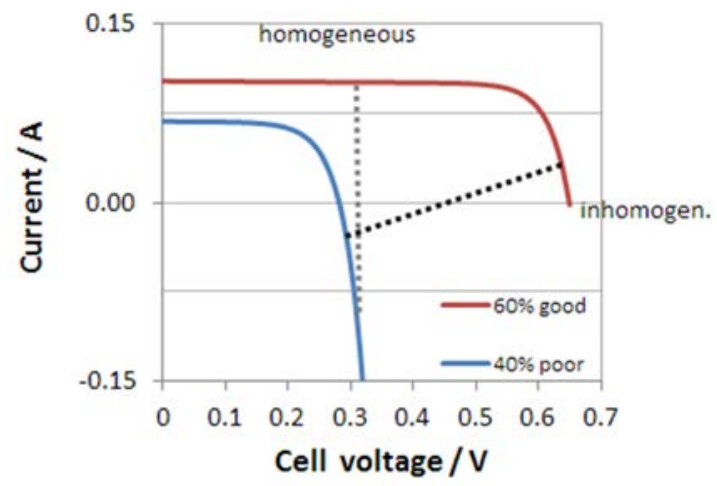

Figure 5: Schematic showing the $I-V$ curves of the good and poor area of the cell. The dotted lines connect points with equal but opposing current, i.e. operating points at open circuit. In the case of inhomogeneities the local cell voltage in the good area can much exceed the local voltage in the poor area.

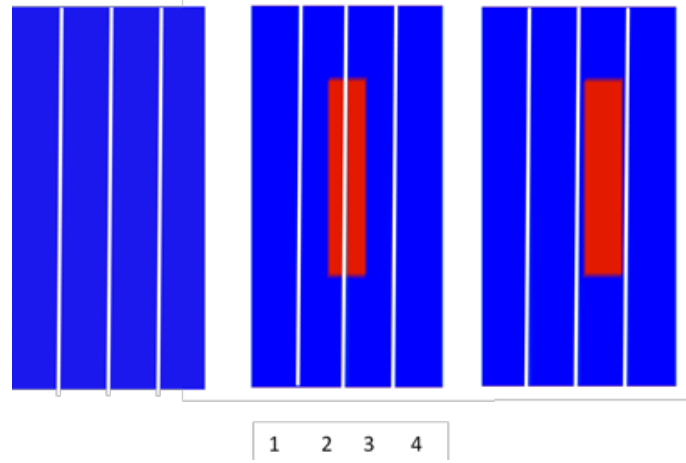

Figure 6: Distribution of the $10 \%$ poor area (red) over 4 cells. Uniformly (left), localized over two adjacent cells(center) and localized within one cell (right). The numbers denote the cell number.

\subsection{Four-cell module}

Calculations were performed for a four-cell module with $10 \%$ poor area. Figure 6 shows the three cases that were compared with the poor area 1) evenly distributed over all cells, 2) localized but divided equally over two adjacent cells, and 3) fully localized in one cell. This latter cell then corresponds to the cell with localized poor area discussed in the previous section. The output of the module is very different for these three cases as the $I-V$ characteristics calculated using the $2 \mathrm{D}$ model with parameters from Table I show (Figure 7). The $V_{o c}$ value increases from $1.4 \mathrm{~V}$ in the distributed case to $2.1 \mathrm{~V}$ in the case of the poor area localized on two adjacent cells and to $2.3 \mathrm{~V}$ in the case of the poor area localized on one cell. The module maximum power output values for these cases are 137, 220 and $261 \mathrm{~mW}$, respectively, which means almost a doubling going from the distributed case to the fully localized case.

Figure 8 shows the contribution of each the of cells to the total output of the module at the MPP. As expected, cells without poor areas contribute most to the overall module output. The series resistance of the monolithic interconnect is in the order of $\mu$-ohms and is therefore negligible. As a result the output of the good, homogeneous cells is not affected by an inhomogeneity in an adjacent cell.

Notice, that according to Figure 8 the output of a poor cell in a module does not strongly depend on the fraction of area affected. Compared to the good cells, all

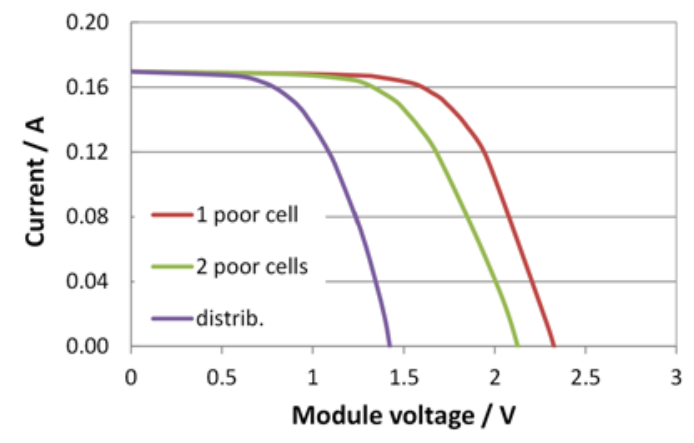

Figure 7: $I-V$ curves of four-cell module with $10 \%$ poor area uniformly distributed over all cells or localized in 2 or one cell, cf. Figure 6. 


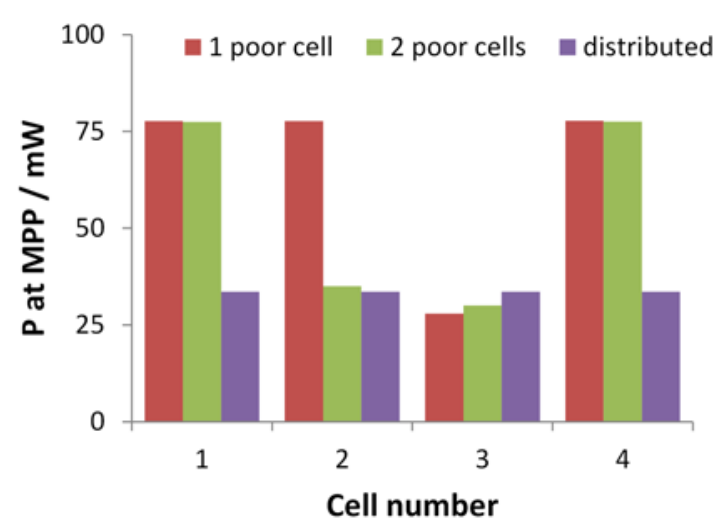

Figure 8: Contribution of the four cells to the power outpout at the maximum power point of the module.

poor cells have a similar lower output although the affected area varies from $10 \%$ in the case of the distributed inhomogeneity to $20 \%$ in the case of 2 poor cells, and to $40 \%$ in the case of 1 poor cell. The main cause for this is that, in order to achieve the maximum output, the current of the module with good and poor cells will be close to the current at MPP of the good cells. The poor cells are forced to operate at a point beyond their maximum power point. This explains why, when all poor area is localized on it, the contribution of cell 3 to the module output, is less than the maximum power output reported in the previous section.

\section{DISCUSSION}

The analysis presented here shows that the impact of inhomogeneities depends strongly on their location in the module. A very important role must be attributed to the distributed series resistance of the contact layers. The cells as well as the modules try to achieve the maximum power output. The finite conductivity of the contact layers allows for variations in the cell voltage and generated current. The outcome is an optimum of increased generated power counterbalanced by increased ohmic losses.

When the series resistance of the monolithic interconnect is small, as is the case here, cells do not feel inhomogeneities in the adjacent cell. This allows them to operate close to their MPP and have cells with non-ideal properties take the full penalty. The number of cells affected is then a very important parameter for the module output.

These results also underline the difficulties that may arise with the interpretation of effective parameters extracted by fitting equations for an equivalent circuit based on a limited set of diodes to the experimental data, as already pointed out by Malm and Edoff [5].

The method presented here is quite generic for thin film solar modules, and therefore not limited to CIGS modules but also applicable to other thin film modules. The type of inhomogeneity is not limited to variations in the dark saturation current but can include variations in photogeneration current, shunts as well as variation in the effective conductivity of the contact layers. Furthermore, the method can be extended with a more sophisticated treatment of the monolithic contact, inclusion of a grid on the $\mathrm{ZnO}: \mathrm{Al}$ surface and contact resistances between the active layers and the Mo-contact layer.

\section{CONCLUSIONS}

A 2-dimensional Finite Element Model for CIGS modules was used to predict the impact of cell inhomogeneities on the module performance. The model consists of a 2D network of parallel 1-diode circuits that are coupled by conductive layers. In the present work the interconnect is represented by a small ohmic resistance.

It was shown that if an area with poor diode characteristics is localized within one cell, the effects are mitigated due to shielding. When the poor diode characteristics extend over more than one cell, the module output strongly decreases with the number of cells affected.

It was pointed out that the model can deal with other types of inhomogeneities as well. Moreover, due to the generic way problem is treated, it can also be adapted for other types of series connections of photovoltaic cells.

\section{ACKNOWLEDGEMENT}

Part of this work was carried out in the context of the project "SOPHIA - Solar Photovoltaic European Research Infrastructure". SOPHIA is a project supported by the European Commission in the 7th Framework Programme (GA $\mathrm{n}^{\circ}$ 262533). For further information about SOPHIA see: www.sophia-ri.eu.

\section{REFERENCES}

[1] B. von Roederen, "National Solar Technology Roadmap CIGS PV", NREL/MP-520-41737 (2007).

[2] I. Repins, S. Glynn, J. Duenow, T. J. Couts, W. K. Metzger, and M. A. Contrera, Proc.SPIE 7409 (2009) 74090M.

[3] P. Mack, T. Ott, F. Schwäble, F. Runai, and T. Walter, (2010).

[4] T. Ott, F. R. Runai, F. Schwäble, and T. Walter, Prog.Photovolt: Res.Appl. 20 (2012) 600-605.

[5] U. Malm and M. Edoff, Prog.Photovolt: Res.Appl. 16 (2008) 113-121.

[6] U. Malm and M. Edoff, Prog.Photovolt: Res.Appl. 17 (2009) 306-314.

[7] P. O. Grabitz, U. Rau, and J. H. Werner, Phys.Stat.Sol.(a) 202 (2005) 2920-2927.

[8] K. Brecl, M. Topic, and F. Smole, Prog.Photovolt: Res.Appl. 13 (2005) 297-310. 